University of Louisville

ThinkIR: The University of Louisville's Institutional Repository

\title{
$5-2013$
}

\section{The effect of inversion on infant attention disengagement from}

\section{faces.}

Jessica Pence

University of Louisville

Follow this and additional works at: https://ir.library.louisville.edu/honors

Part of the Child Psychology Commons, and the Cognition and Perception Commons

\section{Recommended Citation}

Pence, Jessica, "The effect of inversion on infant attention disengagement from faces." (2013). College of Arts \& Sciences Senior Honors Theses. Paper 48.

http://doi.org/10.18297/honors/48

This Senior Honors Thesis is brought to you for free and open access by the College of Arts \& Sciences at ThinkIR: The University of Louisville's Institutional Repository. It has been accepted for inclusion in College of Arts \& Sciences Senior Honors Theses by an authorized administrator of ThinkIR: The University of Louisville's Institutional Repository. This title appears here courtesy of the author, who has retained all other copyrights. For more information, please contact thinkir@louisville.edu. 
The Effect of Inversion on

Infant Attention Disengagement from Faces

By

Jessica Pence

Submitted in fulfillment of the requirements for

Graduation summa cum laude

and

for Graduation with Honors from the Department of

Psychological and Brain Sciences Honors Department

University of Louisville

March, 25 2013 
The ability to shift one's attention from one stimulus to another is an important component of an infant's attentional system and is crucial to an infant's ability to learn about his or her environment (Colombo, 2001). Since infants are born with minimal motor skills and language abilities, where they focus their attention is an important part of how they view and learn about the world. Infants' attention towards faces, in particular, is critical because when viewing faces, a variety of important information is taken in, including information regarding communication (both verbal and nonverbal), emotions, and social behavior, among many other things. In fact, it has been argued that the fact that children with autism do not attend to faces as often as typically developing infants may impair their social skills development (e.g. Schultz, 2005). For these reasons, it is important that we understand how attention to faces in infants develops.

Typically, researchers acknowledge the whole process of "attention" as having multiple stages. First is what is commonly called the "orienting" stage. This stage has been defined simply as the aligning of attention with a source of sensory input (Posner, 1980). Essentially, this stage involves the initial onset of attention toward a stimulus. Orienting is often measured behaviorally by looking at "first looks" toward a stimulus (Di Girogrio, Turati, Altoè \& Simion, 2012; Gliga, Elsabbagh, Andravizou, \& Johnson, 2009). The second stage is often referred to as the "sustained attention" stage. This stage involves the active focus of attention to a stimulus. It involves subject-controlled processing of the stimulus information (Courage, Reynolds \& Richards, 2006) and is more voluntary and task-driven than the other phases of attention (Colombo, 2002). In behavioral studies with infants, sustained attention is captured by measuring the duration of an infant's gaze at a stimulus. Lastly, the final stage is called "attention termination" or "attention disengagement." This stage involves "inhibiting" or "turning off" 
attention to a stimulus (Blaga \& Colombo, 2006). Disengagement can be assessed behaviorally by measuring the time it takes infants to shift their attention away from a stimulus.

\section{Infants’ Attention to Faces}

One purpose of the current study is to better understand the underlying processes involved in infants' attention to faces. A vast amount of research has been conducted showing that infants, even newborns, demonstrate a preference for looking at faces over non-face stimuli (e.g. Frank, Vul \& Johnson, 2009; Johnson, Dziurawiec, Ellis, \& Morton, 1991). Their interest in faces leads to the question of what are the processes or the underlying mechanisms involved in infants attending to faces?

Recently, researchers have begun forming interest in this question. Di Giorgio et al. (2012) investigated 3-month-olds, 6-month-olds, and adults in their ability to orient to and prefer a face when presented in an array of non-face objects. In terms of orienting, they found the direction of 3 and 6 months olds' first looks were random, while adults' first looks went significantly more often toward faces than non-face objects. They also found that the total number of fixations was greater toward faces in both the adults and 6-month-olds, however, the 3-month-olds did not show a visual preference for the faces. In another recent study, Libertus and Needham (2011) presented a face and a toy simultaneously on a large screen and monitored their looking behaviors. They found that 3-month-olds were not more likely to look toward the face near the beginning of the trials $(1000-2000 \mathrm{~ms})$ than the toy. However, the 5-month-olds were significantly more likely to look toward the face during that time. Finally, in a similar study, DeNicola, Holt, Lambert, \& Cashon (in press) looked at 4- and 8-month-olds and found that the direction of first looks was random across age. Infants significantly preferred to look at faces across age as well. These recent studies show the increased interest in the topic of the 
various stages of attention and their relation toward faces; however they do not address behavioral measures of attention disengagement (but see Courage, Reynolds, \& Richards, 2006 for heart rate measures of attention disengagement; see Peltola, Leppänene, \& Hietanen, 2011; Doi, Tagawa, \& Shinohara, 2010, for research on disengagement from emotional faces). To fully understand the effects of faces on attention, more research on attention disengagement from faces is needed.

\section{Inversion Effect}

In the present study, we aim to investigate infant disengagement and faces. However, unlike previous studies we also aim to investigate the effect of face inversion on the disengagement process. To adults, faces are harder to recognize when inverted (e.g. Valentine, 1988; Yin, 1969). It has been found that inversion also affects how adults process faces, meaning that adults process upright faces differently than inverted faces (e.g. Maurer, Le Grand, \& Mondloch, 2002; Rossion \& Gauthier, 2002).

Research with infants has shown that inversion affects infants' face perception in the second half of their first year of life (e.g. Kestenbaum \& Nelson, 1990; Schwarzer \& Zauner, 2003). In a set of studies investigating the development of holistic processing of upright and inverted faces, Cohen and Cashon (2001) found that 7-month old infants process upright faces holistically, but inverted faces featurally, meaning they are not processing the face as a whole, but rather in its parts. Subsequently, Cashon and Cohen (2004) found that four-month-olds process both upright and inverted faces holistically, suggesting that 4-month-olds are not as affected by inversion of faces as older infants, and their face perception skills for inverted faces are still fairly strong. Ferguson, Kulkofsky, Cashon, and Casasola (2009), replicated this developmental difference testing 4- and 8-month-olds using the same task. Together, these 
findings indicate that the inversion effect on face processing develops between 4 and 7-8 months of life.

The only study that has studied the effect of face inversion on any stage of attention in infants is by Gliga et al. (2009). In this study, researchers investigated whether inversion of faces interferes with 6-month-old infants' orienting response. Infants were presented with an array of 4 or 6 stimuli that consisted of one upright face, one inverted face, and competing non-face, upright objects. They found that infants oriented significantly more toward faces than objects as measured by the direction of first looks. More importantly, there was no difference in the proportion of first looks toward upright compared to inverted faces. However, they did find that upright faces received a greater number of fixations than inverted faces after their first looks, and inverted faces were the target of as few fixations as the non-face stimuli that were presented as well. In sum, they found that in these 6-month-olds, the number of fixations was affected by inverting a face, but their attention-orienting response was not. Although this study did include inverted faces in relation to attention, it did not address the stage of attention disengagement.

\section{The Current Study}

Our current study focuses on how attention disengagement is affected by face inversion, an area that has not yet been investigated. Infant attention disengagement has previously been studied with non-face stimuli using the gap-overlap paradigm (Hood \& Atkinson, 1993). In this paradigm, an infant's gaze is first drawn to a visual stimulus at the center of the screen. Then a competitive stimulus is presented in the visual periphery, which will typically draw the infant's eye away from the central stimulus and toward the peripheral stimulus. In the gap condition, the central stimulus is removed when the peripheral stimulus is added, whereas in the overlap condition the central stimulus will remain present when the peripheral stimulus is presented. The 
critical difference between these conditions is that on overlap trials, attention has to be disengaged from the central fixation, whereas on gap trials, disengagement from fixation is not needed (Colombo, 2001). When measuring disengagement, the overlap condition is used because it requires the subject to remove their attention from one stimulus to another while both are still presented.

The amount of time that elapses from the moment the peripheral stimulus is presented until the moment the participant shifts his or her gaze away from the center stimulus and toward the peripheral stimulus is called the saccade latency. Measuring the infant's saccade latency has been the primary way to determine disengagement (Blaga and Colombo, 2006, Hood and Atkinson, 1993), with shorter latencies signifying faster disengagement and longer latencies signifying slower disengagement. Research thus far shows that the average latency for fixation to move to the peripheral stimulus decreases with age, meaning that older infants are shown to be significantly faster to shift fixation away from the central stimulus (Frick, Colombo, \& Saxon, 1999). Infants have been found to readily disengage their attention from one stimulus to another by four months (Frick, Colombo, \& Saxon, 1999).

In the current study, we investigated infants' ability to disengage their attention from faces to non-face objects is affected by inversion, and if so, whether this inversion effect develops between approximately 4 and 7-8 months. Because of our particular interest is the disengagement process, we employed the overlap task in order to determine whether there are differences in attention disengagement from upright and inverted faces. We compared the latencies when the center face stimulus was presented in an upright versus inverted orientation. If inversion affects disengagement, we would expect the infants to take longer to disengage from an upright face than an inverted face. Based on previous face processing studies (Cashon \& 
Cohen, 2004; Cohen \& Cashon, 2001; Ferguson et al., 2009), we expected to see an effect of inversion on saccade latency only in the older infants and not in the younger age group.

\section{Method}

\section{Participants}

Fourteen full-term infants of ages ranging from 3.38 months to 8.31 months with normal vision and hearing participated in this study. Participants included eight infants between approximately 4-6 months of age $(M=4.13$ mos., $\mathrm{SD}=0.95,3$ male $)$ and six infants approximately 6-8 months of age $(M=7.35$ mos., $\mathrm{SD}=0.92,3$ male). Three additional participants were not included due to fussiness $(n=2)$ and experimental error $(n=1)$. Ten participants identified themselves as Caucasian, one as African American, one as Native Hawaiian, and two declined to respond. Participants were recruited using contact information obtained from the Kentucky Cabinet for Health and Family Services. Families received a letter in the mail, followed by a phone call inviting them to participate in the study. Participation was voluntary, and parents were given an infant t-shirt or bib to thank them from their participation.

\section{Stimuli}

Stimuli consisted of 16 photographs of Caucasian, female faces of undergraduate students at a local university. Photographs were edited to remove all blemishes and jewelry, and 16 commonly recognized objects (mug, plant, vase, sea shells, red crystal rock, bowl of peppers, piece of jewelry, piece of candy, colorful round toy, helmet, men's ties, wallet, fruit, stack of post-it notes, rubix cube, and a bottle of perfume). All stimuli were presented in color. Half of the faces were presented in the upright orientation and half were presented in the inverted orientation. The order in which they were presented was randomized between subjects. Stimuli were presented on a 50" plasma display with a resolution of 1024 by 768 pixels. Faces and 
objects were comparable in size. Mean visual angles were 6.92 degrees by 7.60 degrees for faces, and 7.08 degrees by 5.10 degrees for objects. An attention-getting stimulus in the form of a flashing blue and white checkerboard-patterned ball was presented in-between trials. This attention-getting stimulus was presented in the center of the display and was accompanied by a repetitive dinging sound.

\section{Apparatus}

Stimuli were presented using Paradigm Stimulus Presentation software (Perception Research Systems, 2009). To record the participants' eye movements, we used an Applied Science Laboratories (ASL) R6 eye-tracker (See Figure 1). The eye-tracking camera utilizes an infrared light source that creates reflections off the cornea and pupil, and the ASL software then tracks an infant's gaze by calculating the vector between these reflections. A magnetic headtracker was also used to allow the eye-tracking camera to follow the infant's head movements. The head-tracker consisted of a transmitter located behind the infant and a small receiver attached to an elastic headband near the infant's right temple (see Figure 2, bottom left panel). The information about the infants head movements was relayed to the ASL eye-tracking software to better maintain the location of the eye as the baby moved in space. If the infant either took the headband off, or if it accidentally fell off during the experiment, the experimenter running the eye-tracking software would switch to manual control of the eye-tracking camera.

A second experimenter viewed the experiment through a second camera that is located below the TV screen. The second experimenter used Paradigm software to control the stimulus presentation. While the attention-getter played, the second experimenter would wait until the infant appeared to be focused on the screen, at that time he or she would press the space bar to begin a trial. This process was repeated until 16 trials were successfully carried out. 
Infants were seated in a high chair placed 64" from the display. The eye-tracking camera was positioned 12" away from the infant, between the display and the high chair. An additional conventional camera was positioned below the monitor and was used to help the experimenter in charge of running the paradigm software know when the infant was paying attention. This view was also displayed on the screen that is used by the experimenter in charge of running the eyetracking software (see Figure 2) so that when coding the data at later time, the observer could also monitor the baby's behavior if needed.

\section{Procedure}

To calibrate the eye-tracker, colorful, moving balls paired with a variety of noises (such as clicking or whistling sounds) were presented sequentially to attract the infant's gaze to the top left and bottom right corners of the screen (see Figure 3). This process was repeated as many times as necessary until proper calibration of the eye-tracker was achieved.

Each trial began with the attention-getting stimulus. Once the infant was looking at the center of the screen, an experimenter would press the spacebar to begin the trial. Infants were presented with either an upright or an inverted face in the center of the screen (see Figure 4). Once the infants gaze had attended to the center stimulus from one second (as calculated by the stimulus presentation software), a competitive non-face object would be presented randomly in either the top left, top right, bottom left, or bottom right corner of the screen. If the infant did not look at the center stimulus for at least one second, the peripheral stimulus would not be presented and the trial would be repeated once 10 seconds had elapsed. Trials ended after 5 seconds had elapsed following the presentation of the peripheral stimulus. To measure infants' attention disengagement, we recorded the amount of time that passed from when the peripheral stimulus 
was presented to when the infant made an eye movement away from the center stimulus - this amount of time is called the saccade latency.

Observers coded saccade latency offline using OpenSHAPA 1.11

(www.openSHAPA.org). Saccade latency was defined as the amount of time from when the peripheral stimulus was presented until the infant's gaze first left the face. In OpenSHAPA, experimenters reviewed the video of each participant completing the experiment (see Figure 5). In each trial the experimenter marked time of the presentation of the peripheral stimulus by pressing " 0 " and pressed "." to mark when the infant's gaze initially left the face. Using this software, we also coded the orientation of the face for each trial after we coded the saccade latency, so we could determine if there was an inversion effect in the infants' ability to disengage. The time-stamped data from OpenSHAPA were run through a macro in Microsoft Excel to calculate the saccade latency for each trial for each participant.

To control for anticipatory saccades, trials where saccade latency was under $250 \mathrm{~ms}$ would have been eliminated, however, no trials under $250 \mathrm{~ms}$ occurred. Additionally, trials were eliminated for the following reasons: (1) the infant looked in a direction other than that of the peripheral stimulus $(n=20),(2)$ tracking of the corneal reflection was lost $(n=16),(3)$ the infant never disengaged from the face $(n=7)$. The average number of trials omitted per infant was 3 trials, and the range was 0 to 7 trials. Refer to Table 1 for a chart of the number of omitted trials for each participant.

\section{Results}

Infants' saccade latencies, our measure of disengagement, are illustrated in Figure 6. To determine whether infants' disengagement differed based on face orientation within each age group, we analyzed saccade latencies using Wilcoxon Signed Rank Tests. For young infants, 
median saccade latency was 493.0 milliseconds on upright trials and 524.2 milliseconds on inverted trials. This difference was not statistically significant $(\mathrm{Z}=-0.420, p=0.674, r=-0.15)$. In the older age group, median saccade latency was 436.2 milliseconds on upright trials and 362.7 milliseconds on inverted trials. This difference was also not statistically significant $(Z=-$ 1.153, $p=0.249, r=-0.47$ ). Power analyses conducted in $\mathrm{G}^{*}$ Power 3.1 (Faul, Erdfelder, Buchner, \& Lang, 2009) indicated that post-hoc power in the older group was equal to 0.24 , which is considerably lower than 0.80 , the desired amount of power. A second analysis indicated that in order to achieve power equal to $0.80,22$ participants would be needed in this age group.

To further investigate the relation between age and saccade latencies by orientation, we examined the relations between age in months and saccade latencies on upright and inverted trials using Spearman's Rank Order Correlations. Scatterplots illustrating relations between age and saccade latencies from upright and inverted faces are shown in Figures 7 and 8, respectively. Although we did not find a statistically significant correlation between age and saccade latency for upright faces ( $\rho=-0.302, p=.293$ ), we did find a statistically significant negative correlation between age in months and saccade latency from inverted faces $(\rho=-0.820, p<.001)$. The older the participants, the faster they were able to saccade away from the inverted faces.

\section{Discussion}

The aim of our study was to determine if inversion affects infant attention disengagement from faces and, if so, whether this effect develops between 4 and 8 months. We hypothesized that the younger infants, around approximately 4-6 months of age, would show no difference in their saccade latency from upright and inverted faces, while the older infants, around 6-8 months of age, would be slower to disengage from upright faces than from inverted faces. We predicted this because past research has shown that inversion begins to have an effect on infants' face 
processing around 7-8 months of age (Cashon \& Cohen, 2004; Cohen \& Cashon, 2001; Ferguson et al., 2009; see also Schwarzer \& Zauner, 2003).

Surprisingly, no significant effects of orientation were found at either age, however, this may be due to our small sample size and not having enough power. Upon inspection of Figure 6, it looks as though a difference between upright and inverted faces is emerging in the older age group. More data needs to be collected before any strong conclusions can be made about these findings.

Although we found that the relation between infant age and saccade latency for upright faces was not statistically significant, meaning that infants did not differ significantly in their saccade latency for upright faces no matter what their age, we did find that a statistically significant relation exists between infant age and saccade latency for inverted faces. Specifically, we found that the older the infant, the quicker they would be to saccade away from an inverted face. This also lends support to the idea that with further data collection, a significant difference between upright and inverted faces within the older age will be found.

Our results contribute to our knowledge of infant perceptual narrowing, which is the developmental pattern whereby the infant's perceptual system becomes attuned to the specific input they experience in their surroundings (Cashon \& DeNicola, 2011; Nelson, 2001; Scott, Pascalis, and Nelson, 2007). This idea that infants start out their first year of life with a broader set of "unconstrained" abilities that becomes more finely tuned and specific as they learn what is most important to take in around them is an idea that has been studied in many different ways. For instance, Werker and Tees (2002) investigated infants' ability to discriminate non-native speech in the first year of life. They found that infants can discriminate non-native speech contrasts without relevant experience, and this ability declines over the first year of life due to 
specific language experience. The same results have been found in face recognition; infants' ability to discriminate between two faces is more broadly tuned at 6 months but becomes more narrowly tuned at 9 months (Pascalis, de Haan, \& Nelson, 2002). Our findings relate well to the previously conducted research in this area because we can see that age in months was significantly negatively correlated with infants' average saccade latency when viewing inverted faces, but not significantly correlated when viewing upright faces. Since prior research has shown that saccade latency tends to decrease with age (Frick, Colombo, \& Saxon, 1999), the fact that saccade latency for upright faces was not significantly correlated with age indicating that upright faces are unique stimuli that seem to hold infant attention throughout the first year of life while other stimuli (such as inverted faces) do not. We can infer from these data that the older infants may have learned that upright faces are of more significance and importance than inverted faces, and thus they will spend more time looking at an upright face than an inverted face.

In addition to our small sample size, the limitations of the study include the inability to look at how faces affect disengagement compared to non-face objects, which would help us to make inferences about whether or not infants are processing inverted faces similarly to how the process objects. To look at this, a study would have to include a condition in which an object was presented as the center stimuli. Another idea for further research would be to include adults as participants to look at the differences in saccade latency in infants and adults. It has been found that adults display an orienting bias toward faces (Tomalski, Csibra, \& Johnson, 2009), and they also demonstrate an inversion effect with faces (Yin, 1969). It would be interesting to investigate the differences in attention disengagement from faces in infants and adults. This would help us to better understand the development of face processing and the inversion effect over time. 
The implications of our findings go beyond improving our understanding of typicallydeveloping (TD) infants. They can also help us to understand the differences in the development of infants with autism or Williams syndrome (WS). Unlike TD infants, older children and adults with autism have been found to avoid faces rather than show a preference for them (e.g. Riby et al. 2011). For example, Riby and Hancock (2009) found that children and adults with autism will avoid faces in a picture of a scene with a face embedded in it as well as in a scrambled picture containing a face. On the other hand, individuals with WS have been found to show an attentional bias toward faces that is even stronger than that of TD individuals, such that the participants with WS were found to look much longer at the face than any other part of the scene in the same study mentioned above. The atypical responses to faces in participants with autism and WS show a difference in the way these two groups are processing the information around them. These atypical responses may relate to differences in attention disengagement in the fact that individuals with WS may attend longer to faces because they struggle to disengage from them. Conversely, people with autism may attend less to faces because they struggle to disengage from non-face stimuli. Looking at TD infants' ability to disengage from faces within their first year of life helps lay the groundwork for determining the similarities and differences that are present in how faces are attended to by these three populations. In the future these findings could lead to earlier diagnoses of autism and a better understanding of the relation between attention to faces starting early in life and later social and communicative development in both autism and WS. 


\section{References}

Blaga, O. M., \& Colombo, J. (2006). Visual processing and infant ocular latencies in the overlap paradigm. Developmental Psychology, 42, 1069-1076. doi:10.1037/0012-1649.42.6.1069

Cashon, C. H., \& Cohen, L. B. (2004). Beyond U-shaped development in infants ${ }^{1}$ processing of faces: An information-processing account. Journal of Cognition and Development, 5, 5980.doi:http://dx.doi.org/10.1207/s15327647jcd0501_4

Cashon, C.H., \& DeNicola, C.A. (2011) Is perceptual narrowing too narrow? Journal of Cognition and Development, 12, 159-162

Cohen, L. B., \& Cashon, C. H. (2001). Do 7-month-old infants process independent features or facial configurations? Infant And Child Development, 10, 83-92. doi:10.1002/icd.250

Colombo, J. (2001). The development of visual attention in infancy. Annual Review Of Psychology, 52337-367. doi:10.1146/annurev.psych.52.1.337

Colombo, J. (2002). Infant attention grows up: The emergence of a developmental cognitive neuroscience perspective. Current Directions In Psychological Science, 11, 196-200. doi:10.1111/1467-8721.00199

Courage, M. L., Reynolds, G. D., \& Richards, J. E. (2006). Infants' Attention to Patterned Stimuli: Developmental Change From 3 to 12 Months of Age. Child Development, 77, 680-695. doi:10.1111/j.1467-8624.2006.00897.x

DeNicola, C.A., Holt, N.A, Lambert, A., \& Cashon, C.H. (in press). Attention-orienting and attention-holding effects of faces on 4- to 8-month-old infants. International Journal of Behavioral Development. doi:10.1177/0165025412474751 
Di Giorgio, E., Turati, C., Altoè, G., \& Simion. F. (2012). Face detection in complex visual displays: An eye-tracking study with 3- and 6-month-old infants and adults. Journal of Experimental Child Psychology, 113, 66-77. http://dx.doi.org/10.1016/j.jecp.2012.04.012

Doi, H., Tagawa, M., Shinohara, K., (2010). Gaze direction modulates the disengagement of attention from facial expression in 10-month-olds. Emotion. 10, 278-282

Faul, F., Erdfelder, E., Buchner, A., \& Lang, A.-G., (2009). Statistical power analyses using G*Power 3.1: Tests for correlation and regression analyses. Behavior Research Methods, 41, 1149-1160.

Ferguson, K. T., Kulkofsky, S., Cashon, C. H., \& Casasola, M. (2009). The development of specialized processing of own-race faces in infancy. Infancy, 14, 263-284. doi:10.1080/15250000902839369

Frank, M. C., Vul, E., \& Johnson, S. P. (2009). Development of infants' attention to faces during the first year. Cognition, 110(2), 160-170. doi:10.1016/j.cognition.2008.11.010

Frick, J. E., Colombo, J., \& Saxon, T. F. (1999). Individual and developmental differences in disengagement of fixation in early infancy. Child Development, 70, 537-548. doi:10.1111/1467-8624.00039

Gliga, T., Elsabbagh, M., Andravizou, A., \& Johnson, M. (2009). Faces attract infants' attention in complex displays. Infancy, 14, 550-562. doi:10.1080/15250000903144199

Hood, B., \& Atkinson, J. (1993). Disengaging visual attention in the infant and adult. Infant Behavior and Development, 16, 405-422.

Johnson, M. H., Dziurawiec, S., Ellis, H., \& Morton, J. (1991). Newborns' preferential tracking of face-like stimuli and its subsequent decline. Cognition, 40, 1-19. doi:10.1016/00100277(91)90045-6 
Kestenbaum, R., \& Nelson, C. A. (1990). The recognition and categorization of upright and inverted emotional expressions by 7-month-old infants. Infant Behavior \& Development, 13, 497-511. doi:10.1016/0163-6383(90)90019-5

Le Grand, R., Mondloch, C. J., Maurer, D., \& Brent, H. P. (2003). Expert face processing requires visual input to the right hemisphere during infancy. Nature Neuroscience, 6 , 1108-1112. doi:10.1038/nn1121

Libertus, K., \& Needham, A. (2011). Reaching experience increases face preference in 3-month-old infants. Developmental Science,14, 1355-1364. doi:10.1111/j.14677687.2011.01084.x

Maurer, D., Le Grand, R., \& Mondloch, C.J. (2002). The many faces of face processing. Trends in Cognitive Psychology, 6, 255-260.

Nelson, C. A. (2001). The developmental and neural basis of face recognition. Infant and Child Development,10,3-18.

Pascalis, O., de Haan, M., \& Nelson, C.A. (2002). Is face processing species-specific during the first year of life? Science, 296, 1321-1323

Peltola, M. J., Leppänen, J. M., \& Hietanen, J. K. (2011). Enhanced cardiac and attentional responding to fearful faces in 7 month old infants. Psychophysiology, 48, 1291-1298.

Posner, M. I. (1980). Orienting of attention. The Quarterly Journal of Experimental Psychology. 32, 3-25. doi: 10.1080/00335558008248231

Rossion, B., \& Gauthier, I. (2002). How does the brain process upright and inverted faces?. Behavioral And Cognitive Neuroscience Reviews, 1, 63-75. doi:10.1177/1534582302001001004

Riby, D. M., \& Hancock, P. J. B. (2009). Do faces capture the attention of individuals with 
Williams syndrome or autism? Evidence from tracking eye movements. Journal of Autism and Developmental Disorders, 39, 421-431. doi: 10.1007/s10803-008-0641-z

Schultz, R. T. (2005). Developmental deficits in social perception in autism: the role of the amygdala and fusiform face area. International Journal of Developmental Neuroscience, 23, 125-141.

Schwarzer, G., \& Zauner, N. (2003). Face processing in 8-month-old infants: evidence for configural and analytical processing. Vision Research, 43, 2783-2793. doi:10.1016/S0042-6989(03)00478-4

Scott, L.S., Pascalis, O., \& Nelson, C.A. (2007). A domain-general theory of the development of perceptual discrimination. Current Directions in Psychological Science, 16, 197-201

Tomalski, P., Csibra, G., \& Johnson, M. H. (2009). Rapid orienting toward face-like stimuli with gaze-relevant contrast information. Perception, 38, 569-578

Valentine, T. (1988). Upside-down faces: A review of the effect of inversion upon face recognition. British Journal Of Psychology, 79, 471-491. doi:10.1111/j.20448295.1988.tb02747.x

Werker, J.F., \& Tees, R.C. (1984). Cross-language speech perception: Evidence for perceptual reorganization during the first year of life. Infant Behavior and Development, 7, 49-63

Yin R.K. (1969). Looking at upside-down faces. Journal of Experimental Psychology,81,141145 
Table 1

Number of Trials Omitted Per Participant Per Reason

\begin{tabular}{llllll}
\hline & & \multicolumn{3}{c}{ Trials } \\
\cline { 3 - 5 } Participant & Age Group & $\begin{array}{l}\text { Never Looked } \\
\text { Away From Face }\end{array}$ & $\begin{array}{l}\text { Looked in Different } \\
\text { Direction }\end{array}$ & $\begin{array}{l}\text { Lost Infant's } \\
\text { Gaze }\end{array}$ & Total \\
\hline $\mathbf{1}$ & Young & 0 & 4 & 1 & $\mathbf{5}$ \\
$\mathbf{2}$ & Young & 0 & 1 & 3 & $\mathbf{4}$ \\
$\mathbf{3}$ & Young & 0 & 2 & 0 & $\mathbf{2}$ \\
$\mathbf{4}$ & Young & 1 & 1 & 0 & $\mathbf{2}$ \\
$\mathbf{5}$ & Young & 1 & 1 & 2 & $\mathbf{4}$ \\
$\mathbf{6}$ & Young & 0 & 2 & 0 & $\mathbf{2}$ \\
$\mathbf{7}$ & Young & 0 & 0 & 1 & $\mathbf{1}$ \\
$\mathbf{8}$ & Young & 0 & 4 & 0 & $\mathbf{4}$ \\
$\mathbf{9}$ & Old & 0 & 0 & 2 & $\mathbf{2}$ \\
$\mathbf{1 0}$ & Old & 1 & 0 & 2 & $\mathbf{7}$ \\
$\mathbf{1 1}$ & Old & 4 & 0 & 3 & $\mathbf{0}$ \\
$\mathbf{1 2}$ & Old & 0 & 0 & 0 & $\mathbf{2}$ \\
$\mathbf{1 3}$ & Old & 0 & 3 & 0 & $\mathbf{4}$ \\
$\mathbf{1 4}$ & Old & 0 & 2 & 2 & \\
\hline \multirow{2}{*}{ Total } & & $\mathbf{7}$ & $\mathbf{2 0}$ & $\mathbf{1 6}$ & \\
\hline
\end{tabular}




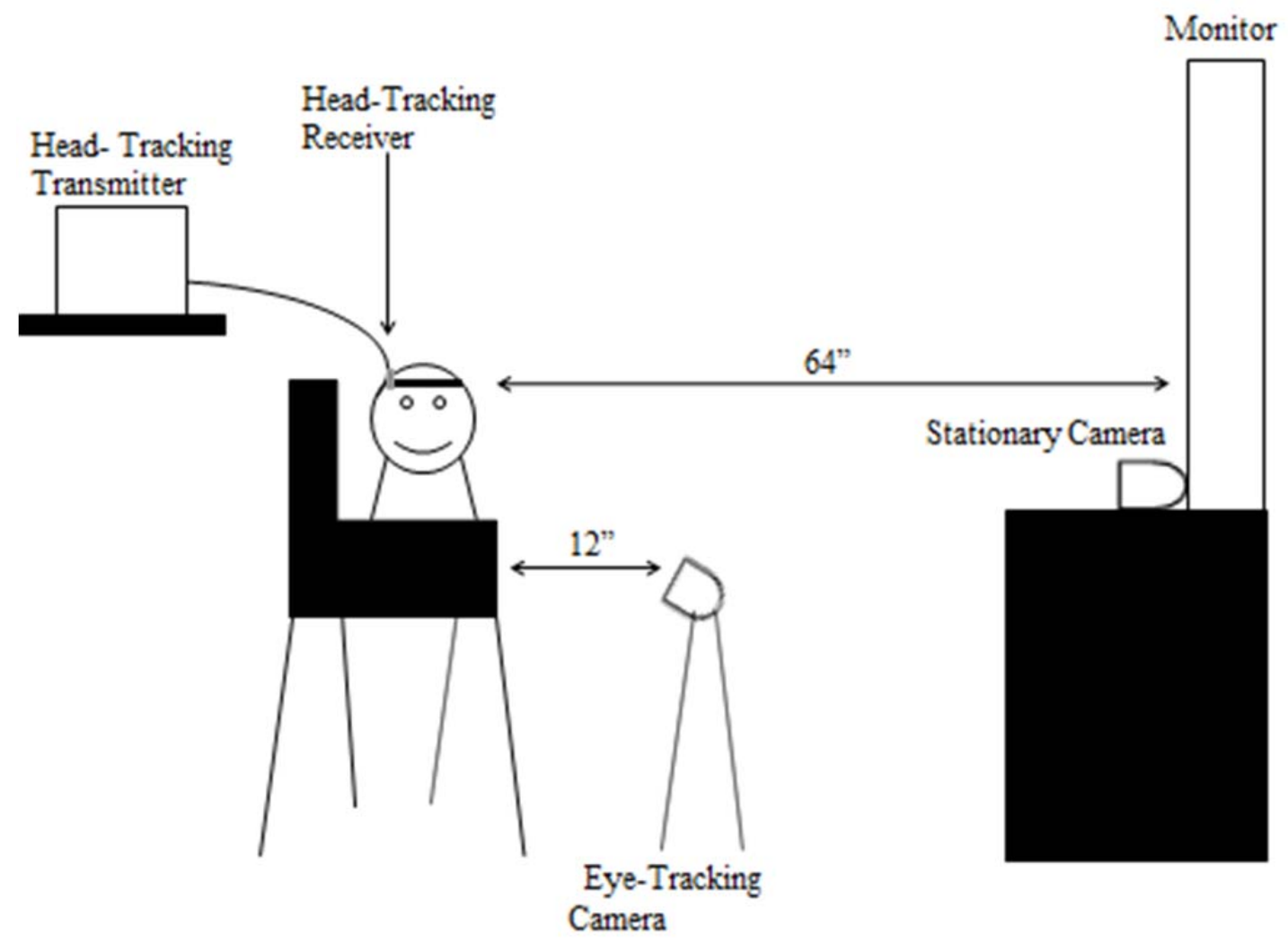

Figure 1. The set-up of the experiment room included the plasma monitor places 64" away from the infant, with the stationary camera placed right in from of the monitor on a table. The eyetracking camera was placed 12" away from the infant, and used the information from the headtracking receiver and transmitter to follow the infant's head movements. 


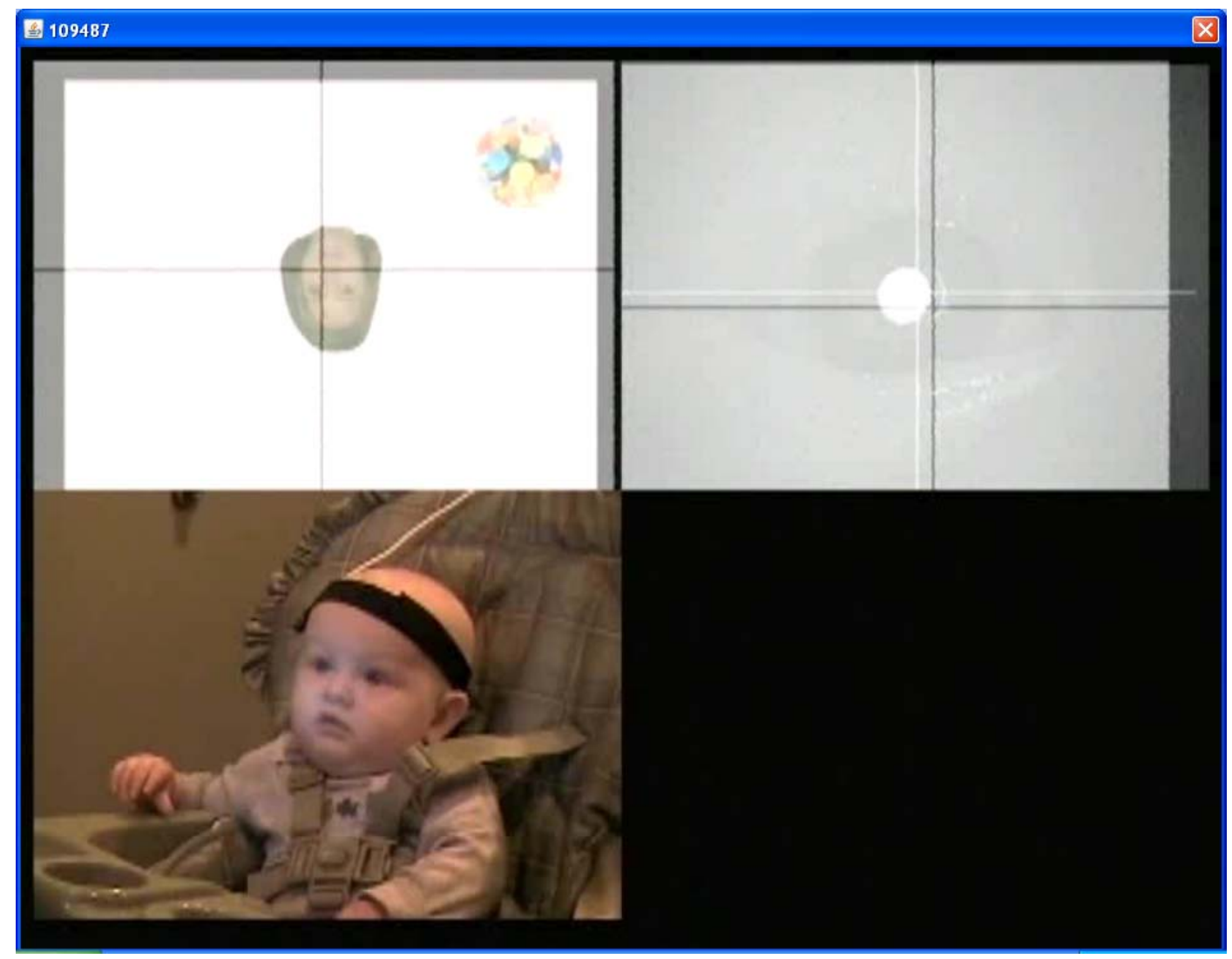

Figure 2.The display that the experimenter running the eye-tracking software uses to calibrate the eye-tracker and determine the location of the eye during the experiment from the image in the top right corner. This display is also used when coding each participant's data by looking at the crosshairs of the infants gaze from the image in the top left corner. 


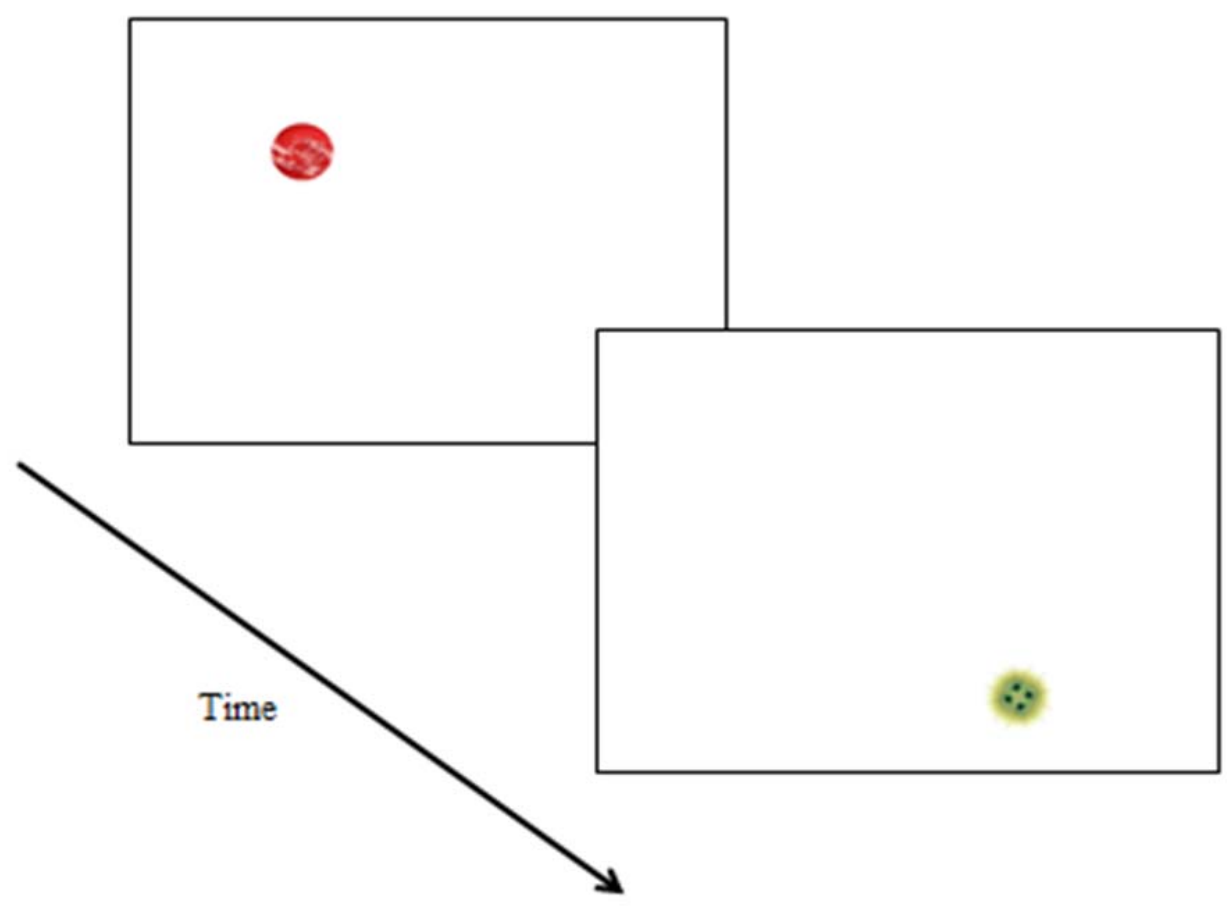

Figure 3. A two-point calibration procedure preceded the experiment for each infant. Eyetracking software recorded pupil and corneal reflections in the top left, and bottom right corners of the screen, and used that data to extrapolate calibration for the rest of the screen. 


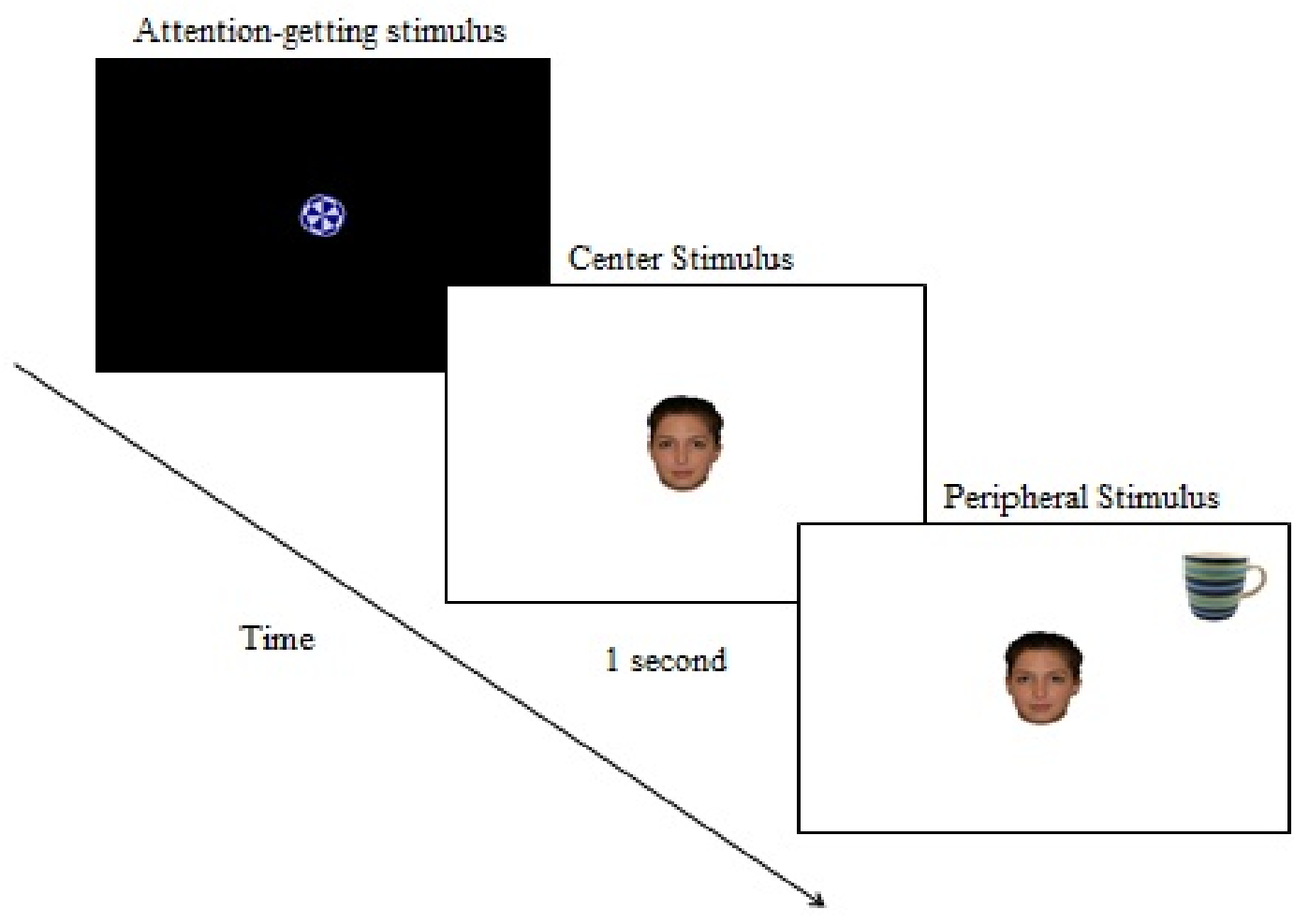

Figure 4. For each trial, the infant was first shown the attention getting stimulus to attract their gaze to the screen. This was followed by the presentation of the center stimulus of a face in either the upright or inverted position. Once the infant looked at the center stimulus for 1 second, the peripheral stimulus would be displayed, while the center stimulus remained on the screen. 


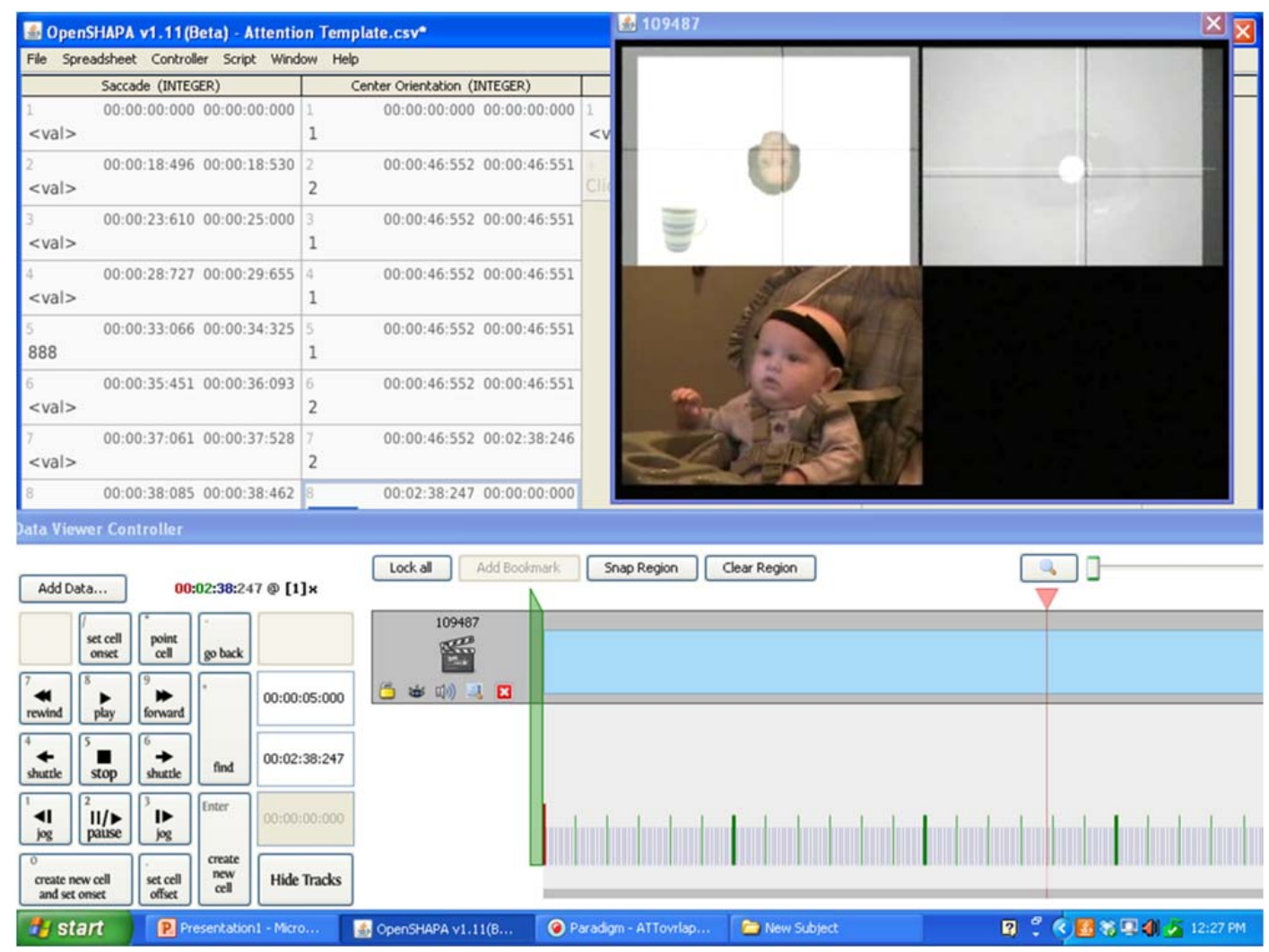

Figure 5. Participant videos were reviewed and coded through OpenSHAPA, which allowed for the coder to place time stamps on the moment in which the peripheral stimulus was presented and the moment that the infant's saccade first left the face. The coder also labeled which trials presented an upright face and which trials presented an inverted face. 


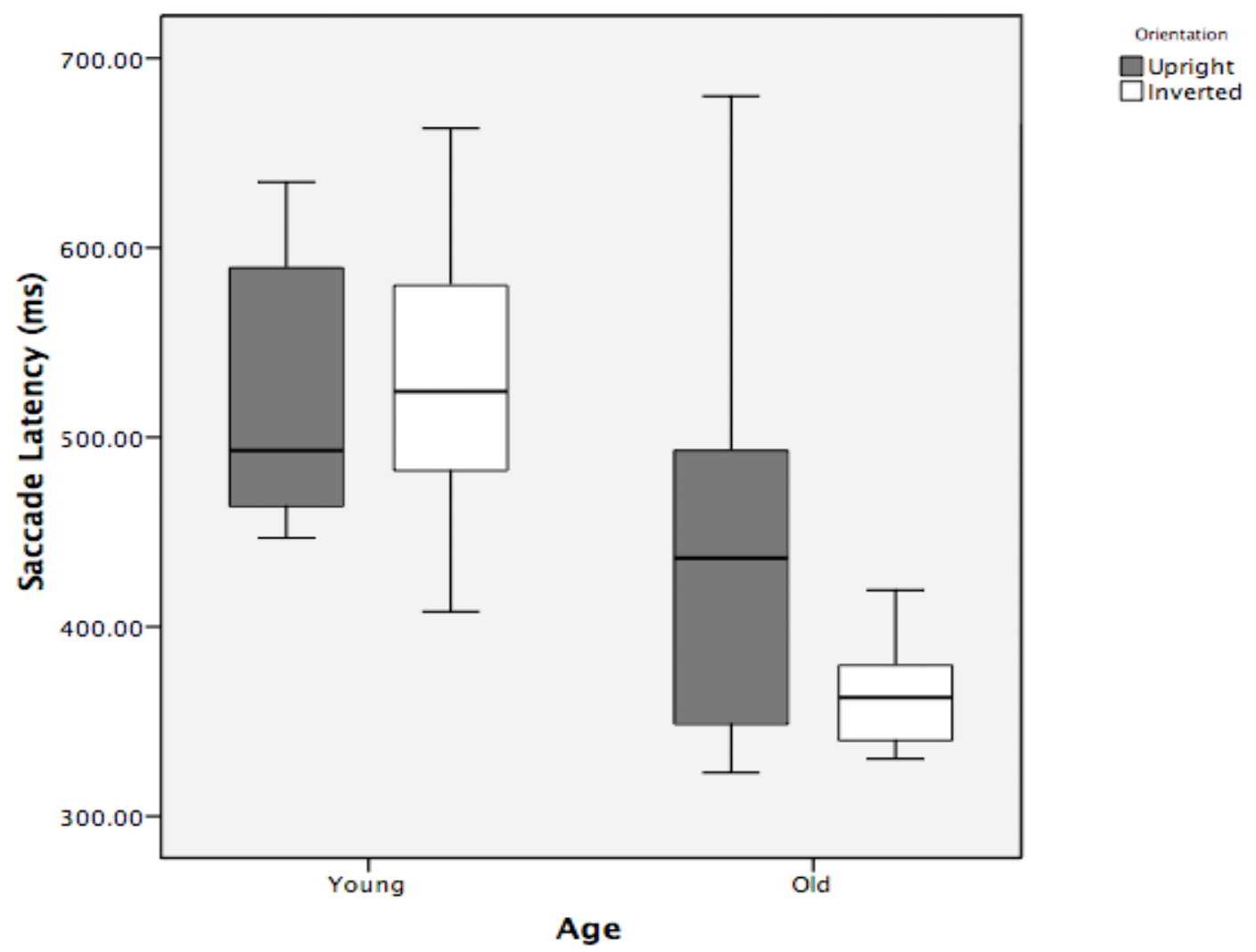

Figure 6. Box plots representing looking times during upright and inverted test trials. The bottom of the box represents the $25^{\text {th }}$ percentile $(\mathrm{Q} 1)$, the center line the $50^{\text {th }}$ percentile, and the top line the $75^{\text {th }}$ percentile $(\mathrm{Q} 3)$. 


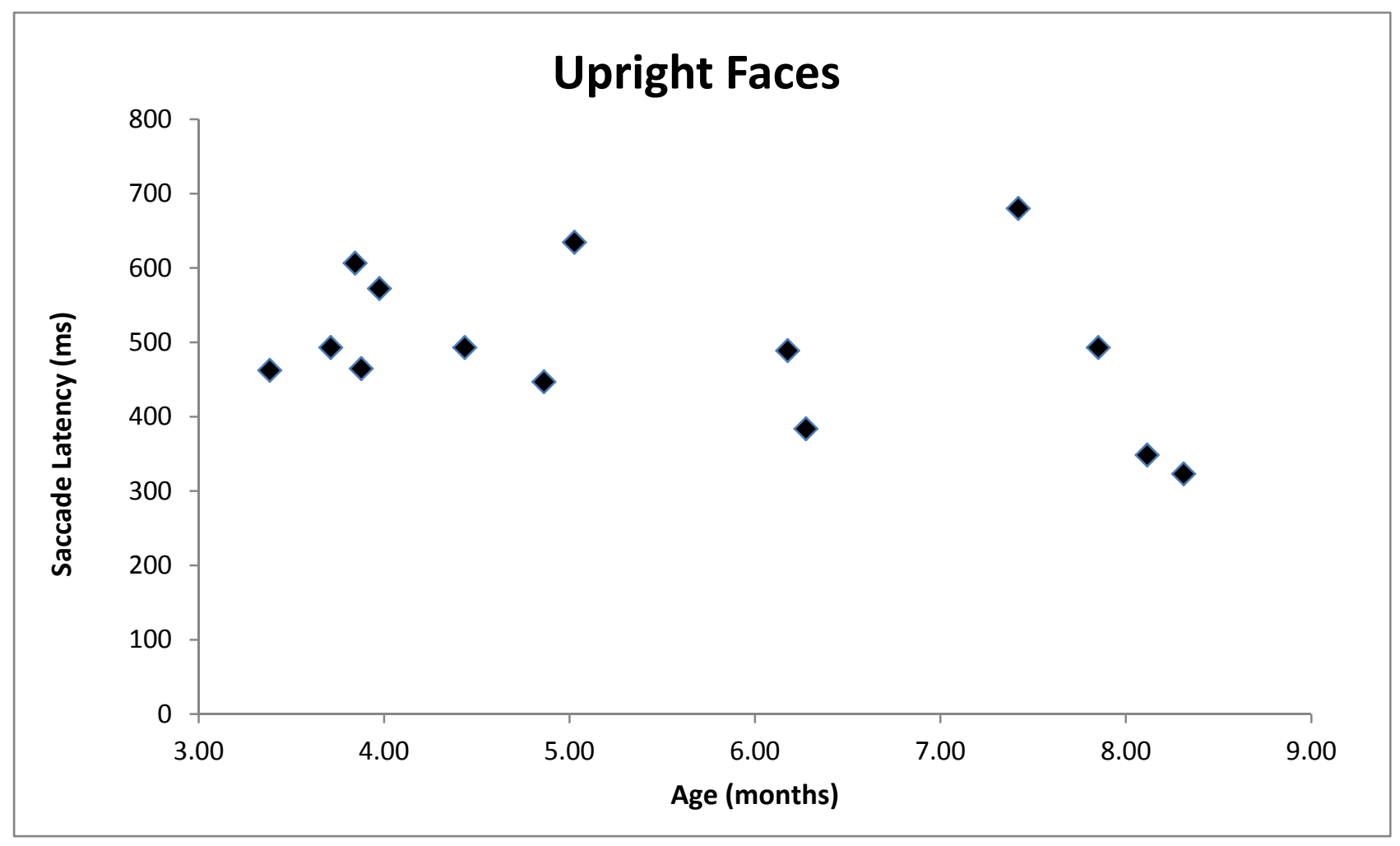

Figure 7. Correlation representing the relation between the age in months of the participant and their mean saccade latency from upright faces. These results were not statistically significant. 


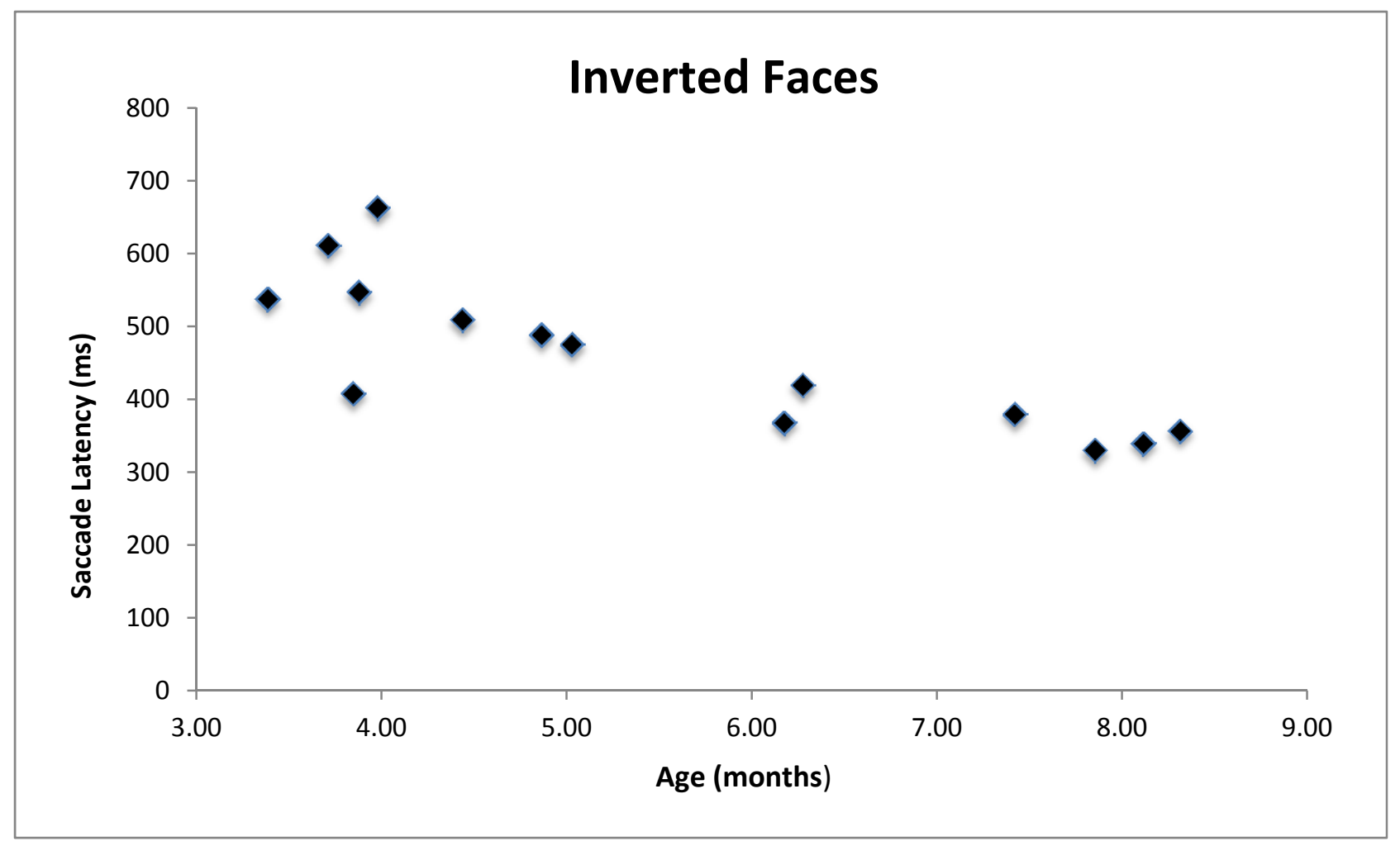

Figure 8. Correlation representing the relation between the age in months of the participant and their mean saccade latency from inverted faces $(\rho=-0.820, p<.001)$. These results were statistically significant. 